2012-01-01

\title{
Simulation and Analysis of Stochastic Signals using the Kolmogorov-Feller Equation
}

Jonathan Blackledge

Technological University Dublin, jonathan.blackledge@tudublin.ie

Marc Lamphiere

Kieran Murphy

Technological University Dublin

See next page for additional authors

Follow this and additional works at: https://arrow.tudublin.ie/engscheleart2

\section{Recommended Citation}

Blackledge, J. et al. (2012) Simulation and Analysis of Stochastic Signals using the Kolmogorov-Feller Equation. Irish Signals and Systems Conference, 2012, NUI Maynooth, 2012.doi:10.1049/ic.2012.0168

This Conference Paper is brought to you for free and open access by the School of Electrical and Electronic Engineering at ARROW@TU Dublin. It has been accepted for inclusion in Articles by an authorized administrator of ARROW@TU Dublin. For more information, please contact arrow.admin@tudublin.ie, aisling.coyne@tudublin.ie,gerard.connolly@tudublin.ie.

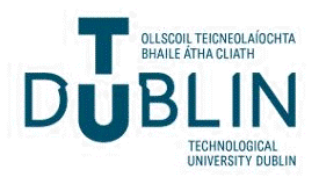


Authors

Jonathan Blackledge, Marc Lamphiere, Kieran Murphy, Shaun Overton, and Afshin Panahi

This conference paper is available at ARROW@TU Dublin: https://arrow.tudublin.ie/engscheleart2/48 


\title{
Simulation and Analysis of Stochastic Signals using the Kolmogorov-Feller Equation
}

\author{
Jonathan Blackledge, Marc Lamphiere, Kieran Murphy, Shaun Overton and \\ Afshin Panahi \\ Computational Finance Research Group \\ Dublin Institute of Technology, Kevin Street, Dublin 8, Ireland
}

\begin{abstract}
We consider a Green's function solution to the Classical KolmogorovFeller Equation which requires an iterative approach for which a sufficient convergence condition is derived. The solution obtained is applied to the simulation of signals whose spectral properties are determined by a Characteristic Function of the form $|k|^{-q}, \quad q>$ 0 where $k$ is the spatial frequency and $q$ is the 'Fourier Dimension'. Using example macroeconomic financial time series (FTSE Close-of-Day and the Dow Jones Industrial Average), correlations are observed to exist between $q$ and long term trends in the series using a standard moving window process. This result provides the potential for developing an accurate short term forecasting strategy.
\end{abstract}

Keywords - Stochastic signal modelling, the Kolmogorov-Feller equation, financial time series analysis

\section{INTRODUCTION}

The diffusion-type and jump-type properties associated with the Kolmogorov-Feller Equation (e.g. [1]-[4] and references therein) make it suitable for modelling stochastic signals with long term correlations or 'trends' including financial time series, for example. However, surprisingly there is relatively little published material on the KolmogorovFeller Equation (KFE) and its applications, particularly with regard to solutions appropriate for simulating and analysing stochastic signals. A short overview is therefore given in this paper on the derivations of the Classical and Generalised KFE [5]-[10].

A Green's function solution to the (Classical) $\mathrm{KFE}$ is then considered which provides an expression for the Finite Impulse Response function of a system described by the KFE. This is the principal focus of the paper and reflects an original contribution to the field. A specific solution is then considered based on the application of a Characteristic Function of the form $|k|^{-q}, \quad q>0$ where $k$ is the spatial frequency and some example simulations provided. The inverse problem associated with computing the index $q$ for a given input is then considered and applied to financial time se- ries analysis on a moving window basis. It is shown that a correlation exists between the trending behaviour of a time series and $q$ which is demonstrated using FTSE (Close-of-Day) and Dow Jones (Weekly) Industrial Average data.

\section{The Classical and Generalised KFE}

For a Probability Density Function (PDF) $p(x)$ with Characteristic Function (i.e. Fourier transform) $P(k)$, the 'evolution equation' for the 'Density Field' $u(x, t)$ is given by[11]

$$
u(x, t+\tau)=u(x, t) \otimes_{x} p(x)
$$

where $\otimes_{x}$ denotes the convolution integral over $x$. The Density Field represents the evolution in time $t$ of a concentration of particles over a length $x$ undergoing a random walk process in a period of time $\tau$ which conforms to the PDF $p(x)$.

Consider the Taylor series for the function $u(x, t+\tau)$, i.e.

$u(x, t+\tau)=u(x, t)+\tau \frac{\partial}{\partial t} u(x, t)+\frac{\tau^{2}}{2 !} \frac{\partial^{2}}{\partial t^{2}} u(x, t)+\ldots$

For $\tau<<1$,

$$
u(x, t+\tau)=u(x, t)+\tau \frac{\partial}{\partial t} u(x, t)
$$


and we obtain the 'Classical KFE',

$$
\tau \frac{\partial}{\partial t} u(x, t)=-u(x, t)+u(x, t) \otimes_{x} p(x)
$$

Equation (1) is based on a critical assumption which is that the time evolution of the field $u(x, t)$ is influenced only by short term events and that longer term (historical) events have no influence of the behaviour of the field, i.e. the 'system' described by equation (1) has no 'memory'. This statement is the physical basis upon which we introduce the condition $\tau<<1$ thereby allowing the Taylor series expansion of the $u(x, t+\tau)$ to be made to first order. The question then arises as to how longer term temporal influences can be modelled, other than by taking an increasingly larger number of terms in the Taylor expansion of $u(x, t+\tau)$ which is not of practical analytical value. For arbitrary values of $\tau$,

$$
\begin{gathered}
\tau \frac{\partial}{\partial t} u(x, t)+\frac{\tau^{2}}{2 !} \frac{\partial^{2}}{\partial t^{2}} u(x, t)+\ldots \\
\quad=-u(x, t)+u(x, t) \otimes_{x} p(x)
\end{gathered}
$$

We can model the effect on a solution for $u(x, t)$ of the series on the left hand side of this equation in terms of a 'memory function' $m(t)$ and write

$$
\tau m(t) \otimes_{t} \frac{\partial}{\partial t} u(x, t)=-u(x, t)+u(x, t) \otimes_{x} p(x)
$$

where $\otimes_{t}$ denotes the convolution integral over $t \in$ $[0, \infty)$. This is the Generalised KFE which reduces to the Classical KFE when

$$
m(t)=\delta(t)
$$

Note that for any memory function for which there exists a function or class of functions of the type $\hat{m}(t)$, say, such that

$$
\hat{m}(t) \otimes_{t} m(t)=\delta(t)
$$

then we can write the Generalised KFE in the form

$\tau \frac{\partial}{\partial t} u(x, t)=-\hat{m}(t) \otimes_{t} u(x, t)+\hat{m}(t) \otimes_{t} u(x, t) \otimes_{x} p(x)$

where the Classical KFE is recovered when $\hat{m}(t)=$ $\delta(t)$.

Any temporal solution obtained to the Generalised KFE will depend on the choice of memory function $m(t)$ used. There are a number of choices that can be considered each or which is taken to be a 'best characteristic' of a 'system' in terms of the influence of its time history. For example, the Mittag-Leffler function

$$
m(t)=\frac{1}{\Gamma(1-\beta) t^{\beta}}, \quad 0<\beta<1
$$

where

$$
\hat{m}(t)=\frac{1}{\Gamma(\beta-1) t^{2-\beta}}
$$

given that

$$
\int_{0}^{\infty} \frac{\exp (-s t)}{\Gamma(\beta) t^{1-\beta}} d t=\frac{1}{s^{\beta}}
$$

yields the (time) Fractional KFE.

\section{Solution to the KFE}

We now turn our attention to developing a solution to the Classical KFE, i.e. given equation (1), we seek a solution for $u(x, t)$ given $p(x)$. We require a general solution that allows us to investigate the effect of different PDFs $p(x)$ on the function $u(x, t)$ such that the associated inverse problem is practically viable, i.e. where statistical parameter(s) associated with $p(x)$ can be computed from $u(x, t)$. This condition rules out an approach based on, for example, the transformation of equation (1) into Fourier-Laplace space to obtain a solution of the form

$$
u(x, t)=\frac{1}{4 \pi^{2} i} \int_{c-i \infty}^{c+i \infty} \int_{\infty}^{\infty} \frac{\tau U_{0} \exp (i k x) \exp (s t)}{1+s \tau-P(k)} d k d s
$$

where

$$
P(k)=\int_{-\infty}^{\infty} p(x) \exp (-i k x) d x
$$

and

$$
U_{0}(k)=\int_{-\infty}^{\infty} u(x, t=0) \exp (-i k x) d x
$$

which requires an initial condition $u(x, t=0)$ to be stated. Thus, we consider a Green's function solution to equation (1) which we write in the form

$$
\tau \frac{\partial}{\partial t} u(x, t)+u(x, t)=h(x, t)
$$

where

$$
h(x, t)=u(x, t) \otimes_{x} p(x)
$$

The Green's function $g(\tau)$ is then given by the solution of

$$
\tau \frac{\partial}{\partial t} g(\xi)+g(\xi)=\delta(\xi)
$$

where $\xi=t-t_{0}$. Taking the Laplace transform of equations (2) and (3), we have

$$
\tau\left(s \bar{u}-u_{0}\right)+\bar{u}=\bar{h}
$$

and

$$
\tau\left(s \bar{g}-g_{0}\right)+\bar{g}=1
$$


respectively, where

$$
\begin{aligned}
\bar{u}(x, s) & =\int_{0}^{\infty} u(x, t) \exp (-s t) d t, \\
\bar{g}(s) & =\int_{0}^{\infty} g(\xi) \exp (-s \xi) d \xi,
\end{aligned}
$$

$u_{0}=u(x, t=0)$ and $g_{0}=g(\tau=0)$. Form equations (4) and (5), it is clear that

$$
\bar{u}(x, s)=\bar{g}(s) \bar{h}(x, s)
$$

under the condition that $u_{0}=0$ and $g_{0}=0$. Using the convolution theorem for the Laplace transform, equation (6) becomes

$$
u(x, t)=g(t) \otimes_{t} h(x, t)
$$

The Green's function, which is the solution to equation (3), is given by

$$
g(t)=\frac{1}{\tau} \exp (-t / \tau) H(t)
$$

where

$$
H(t)= \begin{cases}1, & t>0 \\ 0, & t<0\end{cases}
$$

From equation (7), it is clear that an iterative solution is required to solve for the function $u(x, t)$ and we therefore consider the iteration, for $m=$ $1,2,3, \ldots$

$$
u_{m+1}(x, t)=g(t) \otimes_{t} u_{m}(x, t) \otimes_{x} p(x)
$$

By transforming equation (8) into Fourier space and using the convolution theorem for the Fourier transform, we can write

$$
U_{m+1}(k, t)=g(t) \otimes_{t} U_{m}(k, t) P(k)
$$

where

$$
U_{m}(k, t)=\int_{-\infty}^{\infty} u_{m}(x, t) \exp (-i k x) d x
$$

and

$$
\begin{gathered}
P(k)=\int_{-\infty}^{\infty} p(x) \exp (-i k x) d x \\
U_{2}(k, t)=g(t) \otimes_{t} U_{1}(k, t) P(k) \\
U_{3}(k, t)=g(t) \otimes_{t} U_{2}(k, t) P(k) \\
=g(t) \otimes_{t} g(t) \otimes_{t} U_{1}(k, t)[P(k)]^{2}
\end{gathered}
$$

and so on for $m=4,5, \ldots, M$. Thus, by induction,

$$
U_{M}(k, t)=\prod_{m=1}^{M} g_{m}(t) \otimes_{t} U_{1}(k, t)[P(k)]^{M}
$$

where

$$
\prod_{m=1}^{M} g_{m}(t) \equiv g(t) \otimes_{t} g(t) \otimes_{t} g(t) \otimes_{t} \ldots
$$

the condition for convergence being given by $\|P(k)\|<\sqrt{2 \tau}$ as shown in Appendix A. We note that the Laplace transform of $g$ is given by

$$
\int_{0}^{\infty} \frac{1}{\tau} \exp (-t / \tau) \exp (-s t) d t=\frac{1}{1+\tau s}
$$

and that, using the convolution theorem for Laplace transforms,

$$
\int_{0}^{\infty} \prod_{m=1}^{M} g_{m}(t) \exp (-s t) d t=\frac{1}{(1+\tau s)^{M}}
$$

Thus, since

$$
\int_{0}^{\infty} \frac{t^{M}}{\tau} \exp (-t / \tau) \exp (-s t) d t=\frac{M !}{(1+\tau s)^{1+M}}
$$

it follows that

$$
\prod_{m=1}^{M} g_{m}(t)=\frac{\exp (-t / \tau) t^{M-1}}{\tau(M-1) !}
$$

Finally inverse Fourier transforming equation (9) and using the convolution theorem for Fourier transforms we can write

$u_{M}(x, t)=\frac{\exp (-t / \tau) t^{M-1}}{\tau(M-1) !} \otimes_{t} u_{1}(x, t) \otimes_{x} \prod_{m=1}^{M} p_{m}(x)$

where it is clear that

$$
\lim _{t \rightarrow 0} u_{M}(x, t)=0 \text { and } \lim _{t \rightarrow \infty} u_{M}(x, t)=0
$$

From equation (10), we define the Impulse Response Function $r(x, t)$ for a 'stochastic system' modelled by equation (1) as

$$
r(x, t)=\frac{\exp (-t / \tau) t^{M-1}}{(M-1) !} \prod_{m=1}^{M} p_{m}(x)
$$

obtained for the case when $u_{1}(x, t)=\delta(x) \delta(t)$. Further, if we define the function

$$
\hat{u}(x)=\int_{0}^{\infty} u_{M}(x, t) d t
$$

then equation (10) can be reduced to the form

$$
\hat{u}(x)=n(x) \otimes_{x} \prod_{m=1}^{M} p_{m}(x)
$$


where

$$
n(x)=\int_{0}^{\infty} \frac{\exp (-t / \tau) t^{M-1}}{\tau(M-1) !} \otimes_{t} u_{1}(x, t) d t
$$

For a $\mathrm{PDF} p(x)$ with Characteristic Function $P(k)$, using the convolution theorem for Fourier transforms, equation (11) transforms to

$$
\hat{U}(k)=N(k)[P(k)]^{M}
$$

\section{Signal Simulation}

Equation (12) is indicative of a standard model for simulating the spectral characteristics of stochastic signals. If we consider the case where $n(x)$ is 'white noise' (whose Power Spectral Density Function (PSDF) is a constant), then the PSDF of $\hat{U}(k)$ is determined by $\left|[P(k)]^{M}\right|^{2}$ and the autocorrelation function of $\hat{u}(x)$ is determined by the inverse Fourier transform of $\left|[P(k)]^{M}\right|^{2}$. Thus, for a Gaussian-type distribution (which defines diffusive processes) given by $P(k)=\exp \left(-k^{2} / M\right)$, for example, we obtain

$$
\hat{u}(x)=n(x) \otimes_{x} \frac{1}{2 \pi} \exp \left[-x^{2} / 4\right]
$$

For a Lévy Characteristic Function given by $P(k)=\exp \left(-|k|^{\gamma}\right)$ where $\gamma \in(0,2]$ is the 'Lévy index' [12]

$$
\hat{u}(x)=n(x) \otimes_{x} \frac{1}{|x|^{1+\gamma}}, \quad x \rightarrow \infty
$$

In this paper, we focus on an application of the 'Characteristic Function' $P(k)=|k|^{-q / M}, \quad q>$ 0 , where $q$ is the Fourier Dimension and when equation (11) becomes [12]

$$
\hat{u}(x)=n(x) \otimes_{x} \frac{1}{\Gamma(q)|x|^{1-q}}
$$

Equation (13) describes a random scaling fractal signal with Fractal Dimension $D$ where $q=(5-$ $2 D) / 2, \quad D \in(1,2)[12]$.

Figure 1 shows examples of signals (rescaled to the range 0-1) simulated using equation (12) with $P(k)=|k|^{-q / M}$ and uniformly distributed 'white noise' for different values of $q$. This result shows the expected effect of increasing the value of the Fourier Dimension on the 'smoothness' of the signal and the longer term trends that emerge as the value of $q$ increases.

\section{Signal Characterisation}

The simulation discussed in the previous section identifies a method of characterising stochastic signals using the Fourier Dimension and further, segmenting such signals using a moving window approach. In the latter case, and based on the ex-
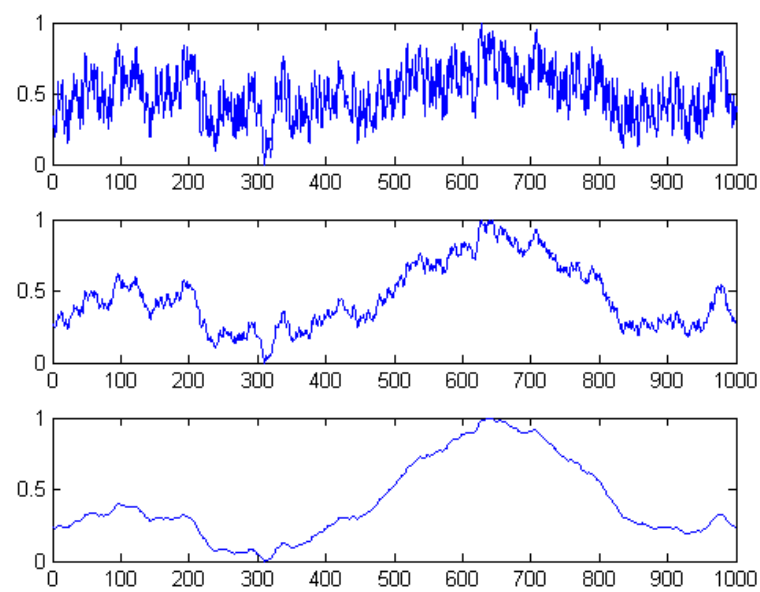

Fig. 1: Example re-scaled signals computed using equation (12) for 'white noise' and Characteristics Function $|k|^{-q / M}$ with $q=0.5$ (top), 1.0 (centre) and 1.5 (bottom).

ample result given in the Figure 1, the Fourier Dimension provides a measure of both the smoothness of a signal and thereby the localisation of correlated features on a non-stationary basis. Nonstationary stochastic signals with significant variations in smoothness and long term correlations include financial time series and in this Section we apply the computation of the Fourier Dimension based on equation (13) to macroeconomic (CloseOf-Day and weekly) signals.

For a function $\hat{u}(x)$ taken over a short period window $x \in[0, W]$, let $n(x) \sim \delta(x)$ in equation (13) so that, ignoring scaling

$$
\hat{u}(x) \sim \frac{1}{|x|^{1-q}}, \quad x \in[0, W]
$$

This is equivalent to the case when $u_{1}(x, t)=$ $\delta(x) u_{1}(t), \quad x \in[0, W]$ in equation (10). Given equation (14), we consider a model for the discrete function $\hat{u}_{n} \equiv \hat{u}\left(x_{n}\right)$ of the form (for $n=$ $1,2, \ldots, N)$

$$
\hat{u}_{n}=a x_{n}^{\alpha}, \quad x_{n}>0
$$

where $a$ is a scaling constant and $\alpha=-(1-q)$. Estimates of the parameters $a$ and $\alpha$ are then chosen to minimise the error function

$$
e(a, \alpha)=\left\|\ln \hat{u}_{n}-\ln u_{n}\right\|_{2}^{2} \equiv \sum_{n=1}^{N}\left(\ln \hat{u}_{n}-\ln u_{n}\right)^{2}
$$

where $u_{n}$ is data which is taken to be normalised, i.e. $\left\|u_{n}\right\|_{\infty}=1$. Differentiating with respect to $A=\ln a$ and $\alpha$ it is trivial to show that

$$
\alpha=\frac{\sum_{n=1}^{N} \ln u_{n} \sum_{n=1}^{N} \ln x_{n}-N \sum_{n=1}^{N} \ln u_{n} \ln x_{n}}{\left(\sum_{n=1}^{N} \ln x_{n}\right)^{2}-N \sum_{n=1}^{N}\left(\ln x_{n}\right)^{2}}
$$


and

$$
a=\exp \left(\frac{\sum_{n=1}^{N} \ln u_{n}-\alpha \sum_{n=1}^{N} \ln x_{n}}{N}\right)
$$

given that

$$
\frac{\partial e}{\partial \alpha}=0 \text { and } \frac{\partial e}{\partial A}=0
$$

Note that in general, $\alpha=1-q$ may be greater than (for $q<1$ ) or less than (for $q>1$ ) zero thereby providing a measure of any (long term) ascending or descending trends in the data $u_{n}$, respective. This effect of illustrated in Figure 2 which shows the result of applying equation (15) to compute $\alpha$ on a moving window basis (using a look-back window of 100 elements) for FTSE Close-of-Day Data [13] after applying a moving average filter (also using a look-back window of 100 elements). Similarly, Figure 3 shows results for the Dow Jones Industrial Weekly Average using look-back windows of 30 elements.

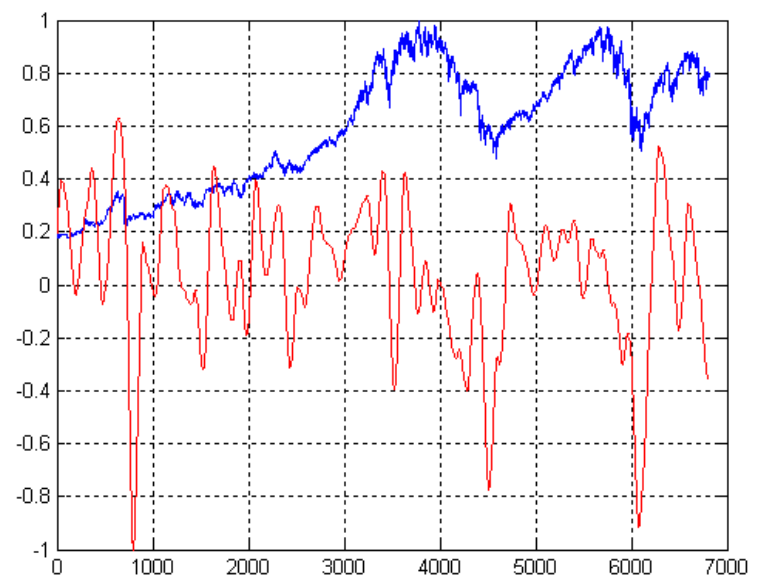

Fig. 2: Normalised FTSE values (close-of-day) from 27-061985 to 03-01-2012 (blue) and normalised values of $\alpha$ given by equation (15) (red) computed using a look-back window of 100 and after applying a moving average filter for a lookback window of 100 .

The sample results shown in Figures 2 and 3 indicates a correlation between the polarity of the signal $\alpha$ and the positive/negative gradient trends associated with the economic index modelled by $\hat{u}$ thereby providing a possible investment and/or forecasting strategy.

\section{Conclusions}

The Kolmogorov-Feller equation provides an approach to modelling density fields characterised by a Probability Density Function $p(x)$ and is a 'statement' of the evolution equation

$$
u(x, t+\tau)=u(x, t) \otimes_{x} p(x)
$$

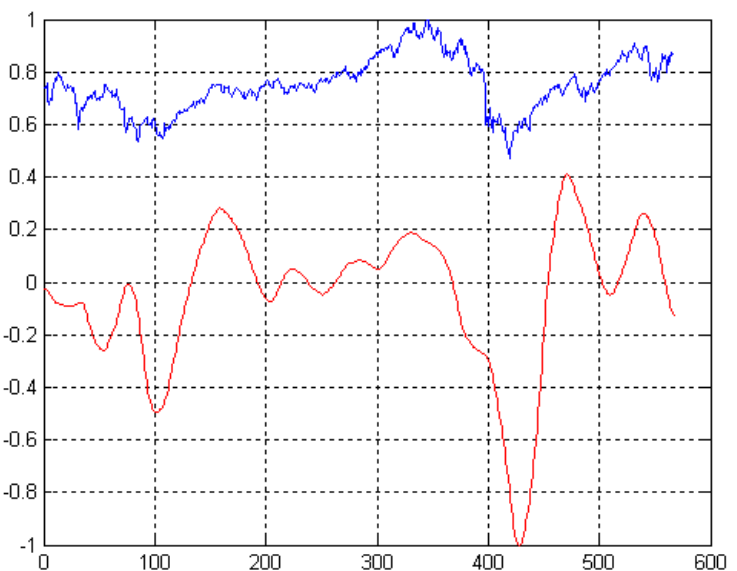

Fig. 3: Normalised Dow Jones Weekly Average from 0708-2000 to 09-01-2012 (blue) and normalised values of $\alpha$ given by equation (15) (red) computed using a look-back window of 30 and after applying a moving average filter for a look-back window of 30 .

under the condition that $\tau<<1$. By introducing a memory function, a generalised form of the equation can be obtained which includes the fractional KFE as discussed in Section II. In this paper, a Green's function solution to equation (1) has been considered and a Finite Impulse Response function derived whose spatial characteristics are determined by multiple convolutions of $p(x)$. By considering the Fourier Dimension associated with a Characteristic Function of the type $|k|^{-q / M}$ it has been shown that the time integrated density field can be written in the form given by equation (13) with Impulse Response Function $|x|^{q-1}$ (ignoring scaling). Analysis of example financial signals using this function as a scaling law for data localised within a moving window reveals a correlation between the direction of a trend and the polarity of $1-q$.

\section{ACKNOWLEDGEMENTS}

Jonathan Blackledge is supported by the Science Foundation Ireland Stokes Professorship Programme

\section{Appendix A: Convergence Condition}

Theorem. A necessary condition for the convergence of the iteration

$$
u_{m+1}(x, t)=g(t) \otimes_{t} u_{m}(x, t) \otimes_{x} p(x)
$$

is $\|P(k)\|<\sqrt{2 \tau}$ where $P(k)$ is the Fourier transform of $p(x)$.

Proof. By taking the Fourier transform of equation (A1), we obtain the iteration

$$
U_{m+1}(k, t)=g(t) \otimes_{t} U_{m}(k, t) P(k)
$$

Let

$$
U_{m}=U+\epsilon_{m} \text { and } U_{m+1}=U+\epsilon_{m+1}
$$


where $\epsilon_{m}$ is the error at iteration $m$. Substituting these equations into equation (A2) we obtain

$$
\epsilon_{m+1}(k, t)=g(t) \otimes_{t} \epsilon_{m}(k, t) P(k)
$$

since

$$
U(k, t)=g(t) \otimes_{t} U(k, t) P(k)
$$

Now consider the operator $\hat{G}=P(k) g(t) \otimes_{t}$ so that we can write

$$
\epsilon_{m+1}(k, t)=\hat{G} \epsilon_{m}(x, t)
$$

and thus

$$
\epsilon_{m}=\hat{G}^{m} \epsilon_{0}
$$

For global convergence, we require that $\epsilon_{m} \rightarrow 0$ as $m \rightarrow \infty$ or

$$
\lim _{m \rightarrow 0} \hat{G}^{m} \epsilon_{0}=0 \quad \forall \epsilon_{0}
$$

Convergence will therefore occur if $\left\|\hat{G}^{m}\right\|<1$ and since $\left\|\hat{G}^{m}\right\| \leq\|\hat{G}\|^{m}$ the condition for convergence becomes

$$
\|\hat{G}\| \leq\|P(k)\| \times\|g(t)\|<1
$$

For Eucliden norms,

$$
\|P(k)\|=\left[\int_{-\infty}^{\infty}|P(k)|^{2} d k\right]^{\frac{1}{2}}
$$

and

$$
\|g(t)\|=\frac{1}{\tau}\left[\int_{0}^{\infty} \exp (-2 t / \tau) d t\right]^{\frac{1}{2}}=\frac{1}{\sqrt{2 \tau}}
$$

Thus, a necessary condition for global convergence is that $\|p(k)\|<\sqrt{2 \tau}$ or $\|p(x)\|<\sqrt{\tau / \pi}$ (using Rayleigh's theorem) which completes the proof.

\section{REFERENCES}

[1] A. N. Kolmogorov, "On Analytic Methods in Probability Theory", Selected Works of A. N. Kolmogorov; Volume II: Probability Theory and Mathematical Statistics (Ed. A. N. Shiryaev), Kluwer, Dordrecht, 1992, 62108,1931 . (Original: Uber die analytischen Methoden in der Wahrscheinlichkeitsrechnung, Math. Ann. 104, 415458, 1931).

[2] J. Neyman, "Proceedings of the Berkeley Symposium on Mathematical Statistics and Probability", Berkeley, California; University of California Press, 403-432, 1949.

[3] W. Feller, "On Boundaries and Lateral Conditions for the Kolmogorov Differential Equations", The Annals of Mathematics, Second Series, Vol. 65, No. 3, 527-570, 1957.
[4] M. Kimura, "Some Problems of Stochastic Processes in Genetics", Annals of Mathematical Statistics, Vol. 28, No. 4, 882-901, 1957.

[5] E. Scalas, R. Gorenflo, F. Mainardi and M. Raberto, "Revisiting the Derivation of the Fractional Diffusion Equation", International Workshop on Scaling in Disordered Systems (Eds. F. Family et al.), World Scientific Publishing, 281-289, 2002.

[6] F. Fan, "A Selective Overview of Nonparametric Methods in Financial Econometrics". Journal on Statistical Science, Vol. 20, No. 4, 317-337, 2005.

[7] F. Mainardia, M. Rabertob, R. Goren and E. Scalasd, "Fractional Calculus and Continuous-Time Finance II: The WaitingTime Distribution", Elsevier, Physica A 287, 468-481, 2000.

[8] R. Jarrow and P. Protter, "A Short History of Stochastic Integration and Mathematical Finance: The Early Years, 18801970", IMS Lecture Notes - Monograph Series, A Festschrift for Herman Rubin (Ed. A. DasGupta) Institute of Mathematical Statistics, 75-91, 2004.

[9] R. Gorenflo, F. Mainardi, M. Raberto and E. Scalas, "Fractional Diffusion in Finance: Basic Theory", A Review Paper Based on a Talk Given by F. Mainardi at MDEF2000 - Workshop 'Modelli Dinamici in Economia e Finanza', Urbino (Italy), September 28-30, 2000 .

[10] S. S. Pai et al. (Eds.), "National Workshop on Fractional Calculus and Statistical Distributions", Proceeding: 25-27 November 2009 at CMS Pala Campus, Kerala, India, Publication No. 41, Centre for Mathematical Sciences Pala Campus, Arunapuram P.O., Pala, Kerala-686 574, India.

[11] A. Einstein, "On the Motion of Small Particles Suspended in Liquids at Rest Required by the Molecular-Kinetic Theory of Heat", Annalen der Physik, Vol. 17, 549-560, 1905.

[12] J. M. Blackledge, "The Fractal Market Hypothesis: Applications to Financial Forecasting", Centre for Advanced Studies, Warsaw University of Technology , ISBN: 978-8361993-01-83, 2010.

[13] Yarhoo Finanace UK \& Ireland, http:// uk.finance.yahoo.com/q/hp?s=\%5EFTSE 